\title{
AN AUTOMATIC AND FAST PROCEDURE FOR THE NUMERICAL ANALYSIS OF CURVED MASONRY STRUCTURES
}

\author{
J. Scacco ${ }^{1}$, G. Milani ${ }^{1}$, and P. B. Lourenço ${ }^{2}$ \\ ${ }^{1}$ Department of Architecture, Built Environment and Construction Engineering ABC \\ Politecnico di Milano, Piazza Leonardo Da Vinci 32, 20133 Milan, Italy \\ E-mail addresses : jacopo.scacco@polimi.it; gabriele.milani@polimi.it \\ ${ }^{2}$ Department of Civil Engineering, ISISE \\ University of Minho, Azurém, 4800-058 Guimarães, Portugal \\ Email address: pbl@civil.uminho.pt
}

Keywords: discrete model, auto-mesh, homogenization, masonry vault.

\begin{abstract}
A fast and innovative discrete model approach coupled with homogenization procedure is here presented. The method is able to comply with the main features required for an accurate simulation of masonry curved elements, such as the orthotropy and the typical inand-out-of-plane coupled behavior exhibited by masonry vaults. Furthermore, homogenization techniques directly implemented in the method allows reducing consistently the number of variables, leading to a fair combination of accuracy and reasonable computational time. The discrete model is an assembly of elastic units joint by non-linear interfaces. These latter are modeled with $3 D$ linear brick elements and Concrete Damage Plasticity (CDP) is used for modeling the non-linear mechanical properties coming from the homogenization step. In order to overcome potential difficulties during the preparation of the model, the discretized mesh is obtained automatically by means of an ad-hoc script implemented by the Authors. The proposed approach is validated taking advantage of numerical data already available on a cloister vault. The numerical comparison shows the reliability of the method and its efficacy in the simulation of both global behavior and crack pattern, requiring a low computational effort.
\end{abstract}




\section{INTRODUCTION}

The vulnerability of historical masonry structures when subjected to seismic loads is well known and preservation is a fundamental task in order to preserve the structures that assumed the role of a cultural landmark. In such historical buildings, the most fascinating and peculiar elements are represented by arches, vaults and domes. However, at the same time, these elements represent often the most vulnerable part of the whole structure. The methodologies according to which curved masonry elements were designed in the past result highly effective for static loads [1]. On the other hand, strong knowledge about the behavior of such elements when subjected to dynamic loads is still missing. From the numerical point of view, the issues are related to the difficulty of balancing reliable results with a reasonable computing time. Several approaches have been developed in literature ranging from methodologies enable to effectively combine ancient and current methods as kinematic limit analyses [2] to approaches that allow a full non-linear analysis. This latter can be faced with both macro-modeling [3] and micro-modeling [4]. The first case leads to an easy numerical implementation simulating the material as a continuum, but the choice of a suitable constitutive model may result cumbersome. On the contrary, a micro-modeling procedure can allow a distinction of all the different constituents but my lead to a huge number of variables and to not practical computing time. These numerical issues can be overtaken by applying homogenization procedures, that ensure a detailed evaluation of the non-linear behavior of masonry at meso-scale $[5,6]$ Such information is then transferred at the structural level in a second step.

In this paper, an innovative and automatic procedure for the non-linear analysis of curved masonry structures is presented.

At the macro-scale level, the structure is described as a repetition of elastic cells joint by interfaces. Such a discrete approach allows reproducing properly the orthotropic behavior of masonry. The non-linear properties coming from homogenization are lumped exclusively at the interfaces, that are modeled as flat brick elements along with a Concrete Damage Plasticity model already available in Abaqus. The main advantage of the method is the direct implementation of the homogenized parameter at the structural level, where even the mesh of the discrete model is created automatically employing a MATLAB script.

The efficacy of the method has been already described for the case of brick walls loaded out-of-plane, in the unreinforced and TRM-reinforced configuration in [7]. The extension of the method even to curved structures is suitable as the modeling of interfaces with flat 3D elements leads to a full coupling of in-and-out-of-plane actions. In such a way the influence of the normal stress related to the gravity loads on the non-linear behavior in flexion is automatically taken into account. The number of variables involved in the numerical simulations is very low when compared to micro approaches, opening the way to further application of the method on reinforced structures.

With the aim to validate the proposed approach, some non-linear simulations have been carried out on a cloister vault, of which experimental and numerical data are available in the literature [8].

\section{METHODOLOGY}

In this section, a general overview of the method is provided. The validation and its implementation are deeply discussed in [7] and the interested readers are directed to this research for a point of reference.

The first main step is the implementation of a discrete mesh of the curved structure. In the case of the wall, such a step resulted straightforward and without complexity due to the simplicity of the geometrical model. On the contrary, the realization of a discrete mesh for vaults 
and domes may lead to a tedious procedure. In fact, the flat 8-noded brick elements employed as interfaces have a thickness that, even though negligible, implies the presence of gaps in the intersections. In order to overcome such limitations, a MATLAB script was implemented by the Authors, enable to provide an automatic discretized mesh. Moreover, the non-linear homogenized properties are assigned automatically to the interfaces according to their orientation. The non-linearity is introduced by taking advantage of the constitutive model Concrete Damage Plasticity (CDP), already available in Abaqus. Its applicability for modeling masonry structures has been widely proved in $[9,10]$ where several non-linear analyses were performed. With such a model, a differential behavior in tension and compression is introduced, following a softening exponential law in the first case and a parabolic softening law in the second one.

Even if CDP is a continuum isotropic constitutive model, its use in a discrete approach allows preserving the orthotropy peculiar to masonry, opening the way to the implementation even in FEM-based software that does not provide an orthotropic constitutive model.

In this paper, a cloister vault, already numerically reproduced in literature, is selected in order to show the capability of the model. The automatic mesh procedure starts with the definition of a rough but structured mesh of the inner surface of the curved structure.

Each 4-noded shell element of the coarse mesh generates correspondent elastic units of the final discrete mesh. In order to create the space for interfaces, each element is scaled by acting on the 3D coordinates of four vertices. Then, the elements are automatically joined with the surrounding ones, generating additional 4-noded narrow elements. These latter will be converted to the non-linear 3D interfaces in the final model. After this stage, each 4-noded element is partitioned, creating a new mesh for the subsequent discrete model.

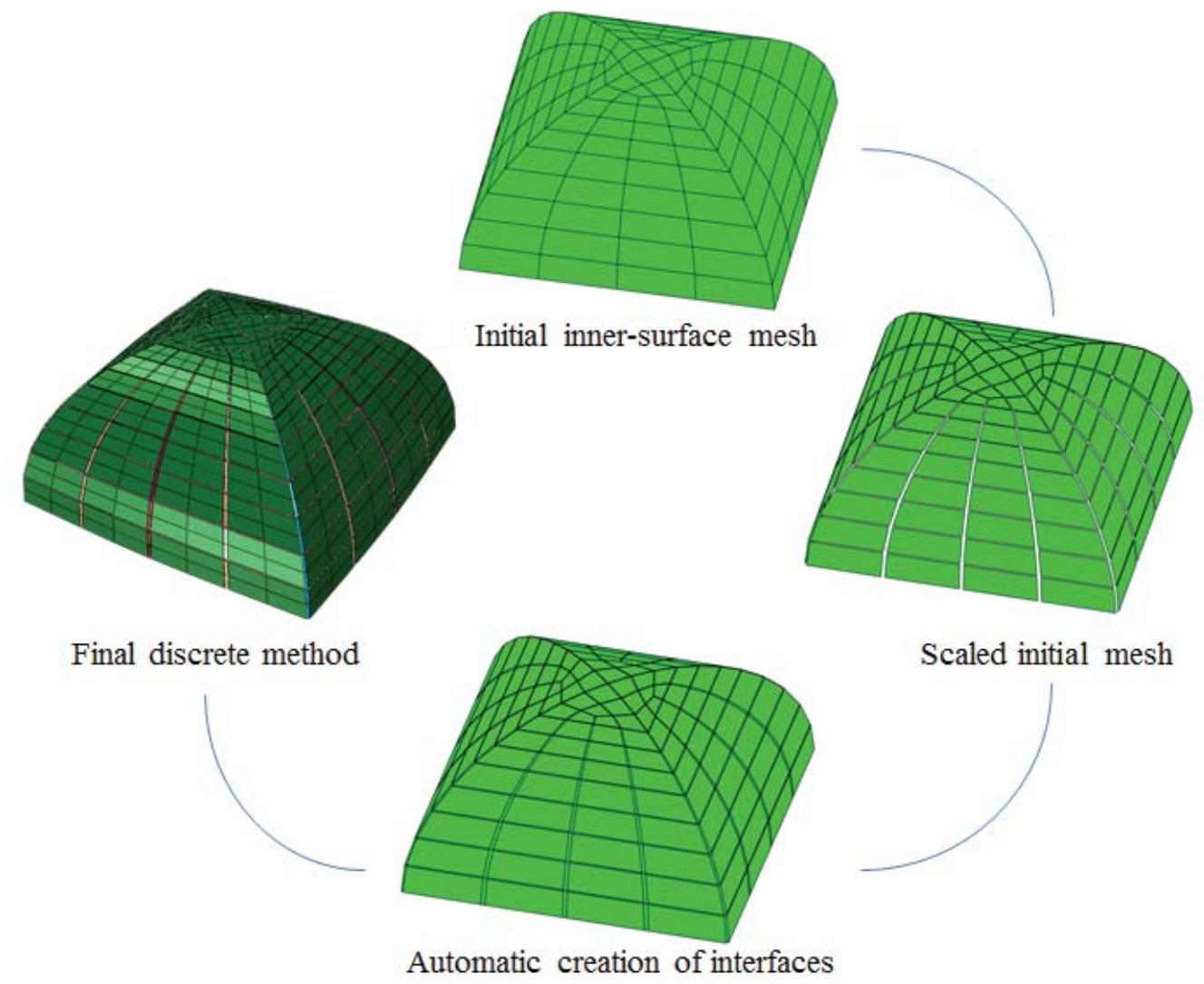

Figure 1: Steps for the automatic implementation of the discrete mesh. 
The final model is then obtained by extrusion of the discrete shell mesh. A different quantity can be applied to each node, making possible the modeling of vaults with variable thickness.

In this work, the cloister vault has been modeled with 160 elastic units having a rough mesh of 4 elements on the plane r-s.
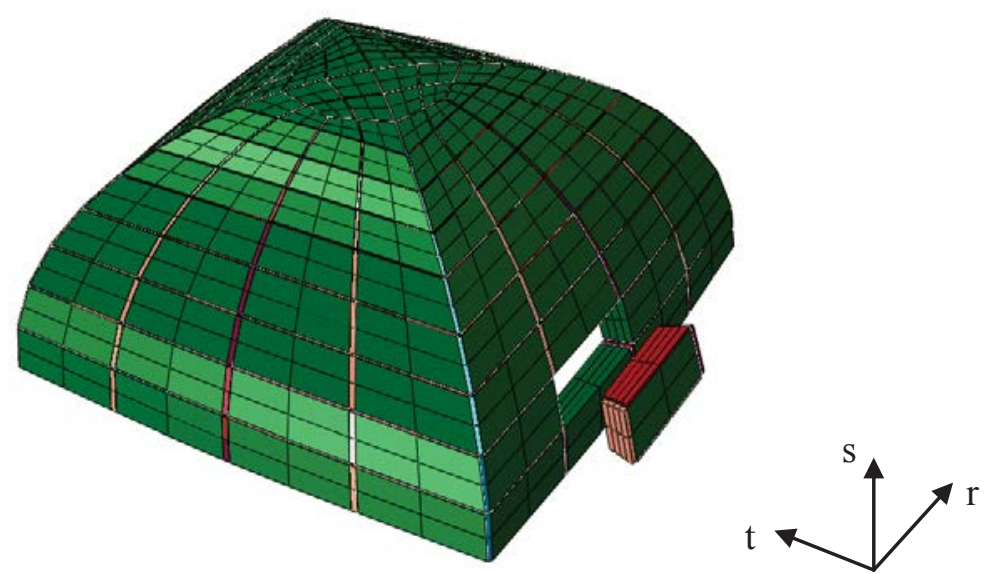

Figure 2: Mesh configuration in detail of the homogenized cloister vault.

According to the experience of the Authors, for reproducing properly the flexural behavior of curved masonry structures, 4 elements along the depth of the dome is a sufficient number.

The possibility to assign automatically the homogenized mechanical properties according to the orientation of the interfaces is a promising starting point for the upcoming research by the Authors. Indeed, semy-analytical approaches [6] might be directly implemented inside the script, leading to a full comprehensive homogenization procedure for curved structures.

Numerical analyses on the cloister vault are available in $[8,12]$. For the sake of consistency, the same homogenized mechanical properties defined in [8] for both directions, are input in the Concrete Damage Plasticity.

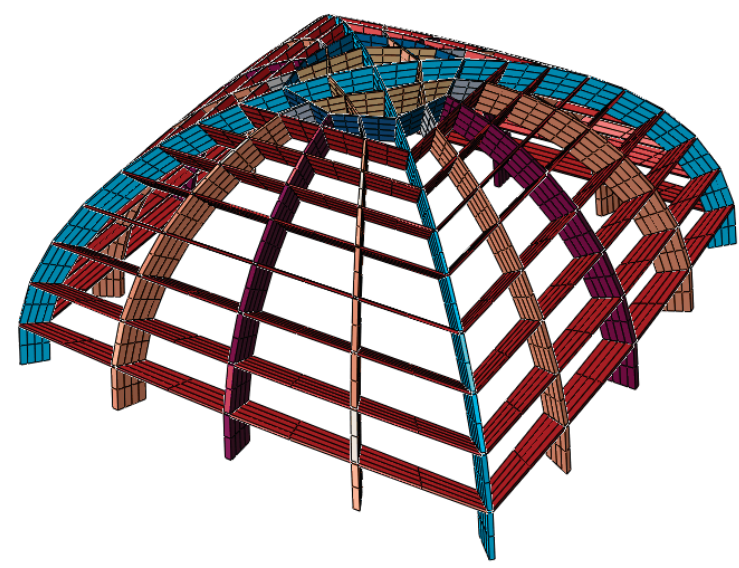

Figure 3: Detail of non-linear interfaces

\section{RESULTS}

The cloister vault, with a square base of $2.2 \mathrm{~m} * 2.2 \mathrm{~m}$, used for the validation of the method was tested by Foraboschi in 2006 and described in [11]. The test consisted in inducing the collapse of the vault by the application of a concentrated force applied at the top. Several nonlinear FE simulations are already available by means of heterogeneous and macroscopic ap- 
proaches. From these latter, a comparison in terms of load-displacement curves is here provided with the proposed method.
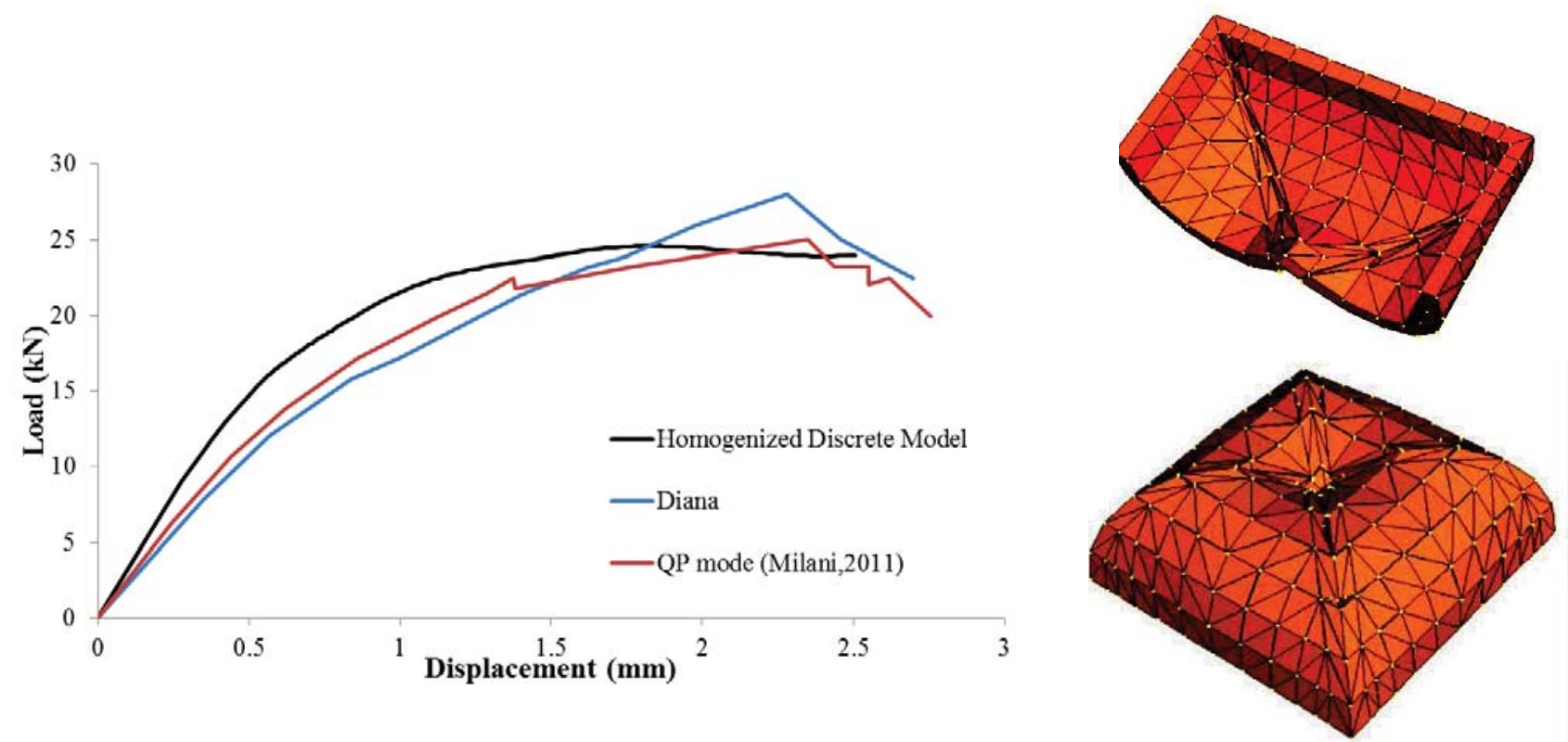

Figure 4: Comparison in terms of load-displacement curves of the proposed method with previous numerical analyses (Left); Collapse mechanism obtained by means of QP non-linear analysis by Milani, 2011 (Right).
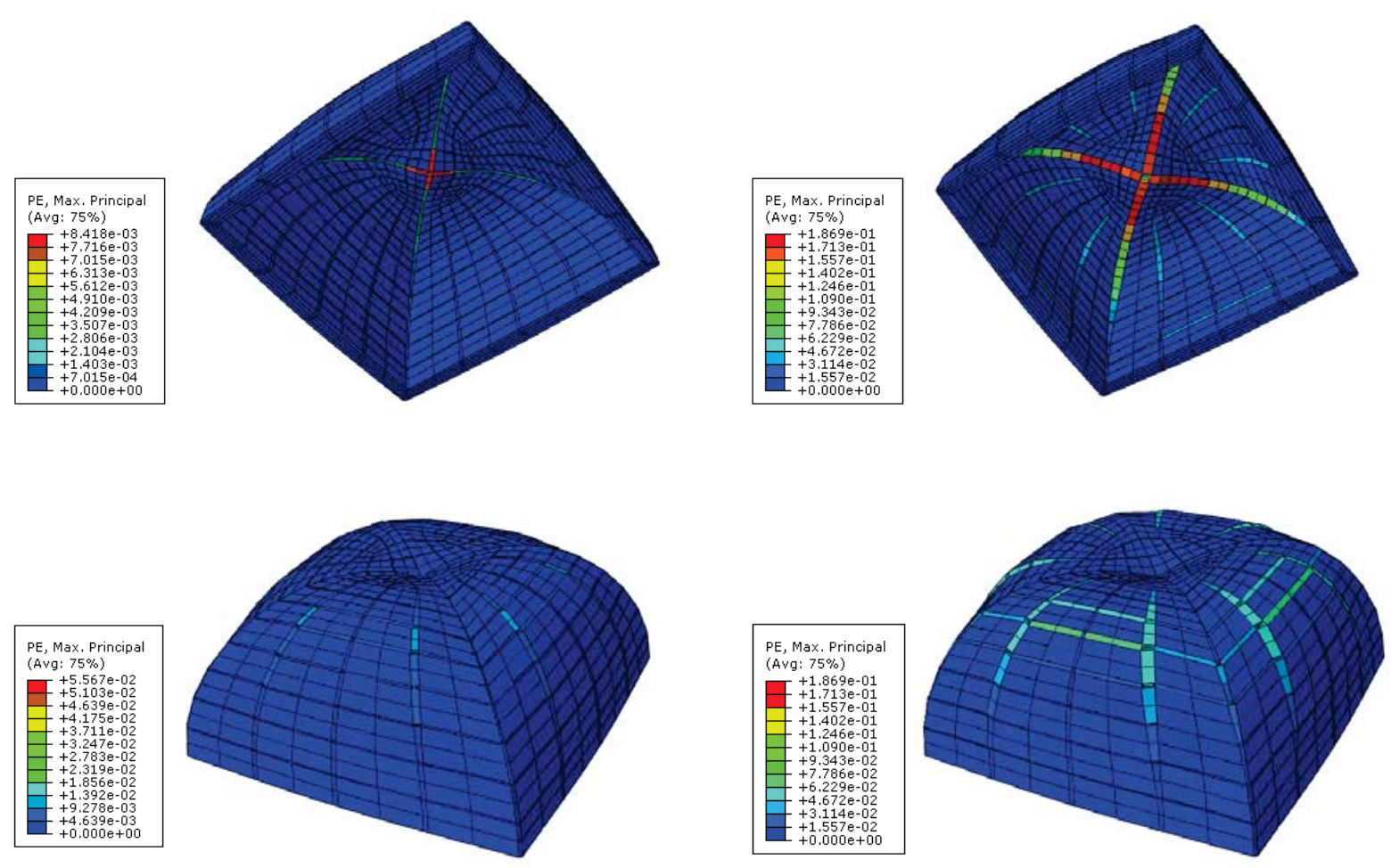

Figure 5: Evolution of crack pattern at the intrados (Top), at the extrados (Bottom).

The global behavior described by DIANA analyses and by other numerical approaches implemented in $[8,12]$ presents a final displacement around $2.7 \mathrm{~mm}$ and a peak load within the range of 25-28 kN. The discrete homogenized approach provides a global behavior that fits 
satisfactorily the numerical references, with a maximum load nearby $25 \mathrm{kN}$ and maximum capacity in displacement around $2.5 \mathrm{~mm}$. The slight difference in the first branch may be attributed to the discrete nature of the method, which does not allow the non-elastic behavior in all the structures but only at the interfaces.

The crack pattern is provided in terms of plastic strains (PE in Abaqus). The cracks start to spread symmetrically along the diagonal at the intrados. Afterward, a circular flexural hinge is determined by the openings at the extrados of the parallels at approximately $2 / 3$ of the height, causing the impossibility to carry further loads.

\section{CONCLUSIONS}

In the present work, a validation of an innovative discrete homogenized method applied on a masonry cloister vault has been provided. First of all, a short overview of already existing methods is discussed to highlight the required features for a new approach and the necessity of implementing it. A discrete method, when complemented with previous homogenization procedures, is presented as the right approach for meeting the following requirements: nonlinear simulation, low-number of variables involved, preservation of orthotropy of material like-masonry, necessity of keeping coupled the in-out-of-plane effects.

The method, already validated on UR and RE out-of-plane-loaded walls, is extended to the case of a cloister vault, experimentally tested in 2006 and numerically simulated several times by different approaches.

The numerical model of the vault is conceived as a repetition of elastic units jointed by non-linear interfaces. These latter are input as flat 8-noded bricks and the non-linearities are introduced by means of the constitutive model Concrete Damage Plasticity, available in the Abaqus library. The discretized mesh is obtained automatically through a MATLAB script implemented by the Authors and the homogenized mechanical properties are, as well, assigned by an automatic procedure to each interface according to their orientation, preserving the orthotropic behavior.

Finally, the homogenized outcome, in terms of load-displacement curves, revealed an excellent capacity to reproduce numerical results coming from more advanced and consuming approaches. Even the evolution of the crack pattern is fully consistent with the information available in the literature.

Moreover, given the low computational burden, it is the opinion of the Authors that interesting extension to reinforced curved elements may be proposed in future works, by applying in a second moment the reinforcement.

\section{REFERENCES}

[1] Huerta S. Mechanics of masonry vaults: the equilibrium approach. Proc. 1st Int. Congr. Struct. Anal. Hist. Constr. Guimaraes, 47-70, 2001.

[2] Grillanda N, Chiozzi A, Milani G, Tralli A. On Collapse Behavior of Reinforced Masonry Domes under Seismic Loads, Key Engineering Materials, 275-82, 2019.

[3] Bianchini N, Mendes N, Lourenco PB. Seismic assessment of masonry cross vaults through numerical seismic assessment of masonry cross vaults through numerical nonlinear static and dynamic analysis, COMPDYN 2019 7th International Conference on Computational Methods in Structural Dynamics and Earthquake Engineering, 2019. 
[4] Bove M, Castellano A, Fraddosio A, Scacco J, Milani G, Piccioni MD. Experimental and Numerical Analysis of FRCM Strengthened Parabolic Tuff Barrel Vault, Key Engineering Materials, 213-20, 2019.

[5] Silva LC, Lourenço PB, Milani G. Derivation of the out-of-plane behaviour of masonry through homogenization strategies: Micro-scale level. Comput. Struct., 209, 30-43, 2018.

[6] Milani G, Bertolesi E. Quasi-analytical homogenization approach for the non-linear analysis of in-plane loaded masonry panels. Constr. Build. Mater., 146, 723-43, 2017.

[7] Scacco J, Ghiassi B, Milani G, Lourenço PB. A fast modeling approach for numerical analysis of unreinforced and FRCM reinforced masonry walls under out-of-plane loading. Compos. Part B Eng., 180, 107553, 2020.

[8] Milani G, Tralli A. A simple meso-macro model based on SQP for the non-linear analysis of masonry double curvature structures. Int. J. Solids Struct., 49, 808-34, 2012.

[9] Valente M, Milani G. Effects of Geometrical Features on the Seismic Response of Historical Masonry Towers E ff ects of Geometrical Features on the Seismic Response of Historical Masonry Towers. J. Earthq. Eng., 1-33, 2017.

[10] Tiberti S, Milani G. Historic City Centers After Destructive Seismic Events, The Case of Finale Emilia During the 2012 Emilia-Romagna Earthquake : Advanced Numerical Modelling on Four Case Studies Abstract : 1059-78, 2017.

[11] P. Foraboschi, Masonry structures externally reinforced with FRP strips: tests at the collapse [in Italian], Proceedings of I Convegno Nazionale "Sperimentazioni su Materiali e Strutture’, 2006.

[12] G. Milani, E. Milani, A. Tralli, Upper bound limit analysis model for FRPreinforced masonry curved structures. Part II: Structural analyses, Comput. Struct. 87 (23-24), 1534-1558, 2009b. 